\title{
Análisis de los resultados del Curso Transversal de Doctorado "Carrera Investigadora", en formato online
}

\author{
Miguel Ferrando Bataller, Marta Cabedo Fabrés y Eva Antonino Daviu \\ Departamento de Comunicaciones. Universitat Politècnica de València \\ mferrand@dcom.upv.es,marcafab@dcom.upv.es evanda@dcom.upv
}

\begin{abstract}
The Communication objectives are to present the results of the implementation of the Massive Online Course of the Doctoral School of UPV called "Research Career" considerig the planning, development and results.

It is a 2 ECT course, taught from year 2014 (3 editions). The course has been designed using the tools provided by the UPV, especially PoliformaT and Polimedia. It has involved an important task of planning and preparation of contents.

The course is structured in 7 teaching units, each unit has 5 Polimedia, supplementary material and a multiple-choice test. The delivery rate has been of one unit per week.

There have been self-assessments of each unit, a discussion forum, work and a final exam. The results of the evaluation and follow-up surveys give good indicators.

This is a new experience for an official doctoral level course with online methodologies. Three year of experience with 91, 110 and 201 students could serve as a real case and implement a model for other courses and Masters degree with similar methodologies.
\end{abstract}

Keywords: Online, PhD, polimedia, poliformaT, mass, distance, multimedia.

\section{Resumen}

Los Objetivos de la comunicación son presentar los resultados obtenidos en la puesta en marcha del Curso Online de la Escuela de Doctorado de la UPV denominado "Carrera Investigadora", desde el punto de vista de planificación, desarrollo y resultados.

Se trata de un curso de 2 créditos ECTS, impartido desde el año 2014 (3 ediciones). El curso se ha realizado utilizando las herramientas facilitadas por la UPV, especialmente PoliformaT y Polimedia. Ha supuesto una tarea importante de planificación y preparación del material.

El curso está estructurado en 7 unidades didácticas, cada unidad cuenta con 5 videos en formato polimedia, material complementario de consulta y un examen. El ritmo de impartición ha sido de una unidad por semana. 
Se han realizado autoevaluaciones de cada unidad, un foro de debate, trabajo y examen final. Los resultados de la evaluación y las encuestas indican un alto seguimiento del curso.

Se trata de un experiencia innovadora para un curso oficial de nivel de doctorado con metodologías online. Tres años de experiencia con $90,110 y$ 201 estudiantes permiten establecer criterios para diseñar otros cursos de nivel de Máster con metodologías similares.

Palabras clave: Online, doctorado, polimedia, poliformat, masivo, distancia, multimedia.

\section{Introducción}

La Universitat Politècnica de València ha impulsado durante los últimos años, dentro de su plan estratégico, la iniciativa "Docencia en Red". Dentro de la iniciativa se ha impulsado la unificación de las Plataformas de Colaboración y Aprendizaje, con el uso generalizado de PoliformaT (Busquets, 2006), la creación de material multimedia de alta calidad (Turró, 2010). Destaca también la definición de los Objetos Digitales de Aprendizaje (Martínez Naharro, 2007).Finalmente las Jornadas de Innovación Educativa y Docencia en Red (Simó, 2014) suponen una oportunidad para presentar los avances basados en estas innovaciones.

La Escuela de Doctorado de la UPV, como estructura responsable de los estudios de doctorado de la Universitat, está ofertado desde el curso 2013-14 un total de 14 cursos de formación transversal, de carácter obligatorio para los alumnos matriculados en los nuevos programas, según la normativa establecida en el RD 99/2011.

\section{Objetivos}

En esta comunicación se presentan los resultados más relevantes de tres años de impartición del Curso Transversal de Doctorado "Carrera Investigadora".

Los Objetivos de la Comunicación son el análisis de los resultados del curso y los ajustes realizados durante los últimos años para la mejora del mismo, partiendo de la experiencia de impartición y los resultados de las encuestas.

El curso tiene una valoración de 2 créditos ECTS y se imparte a lo largo de un período de 8 semanas.

La estructura del curso se muestra en la Fig. 1. 


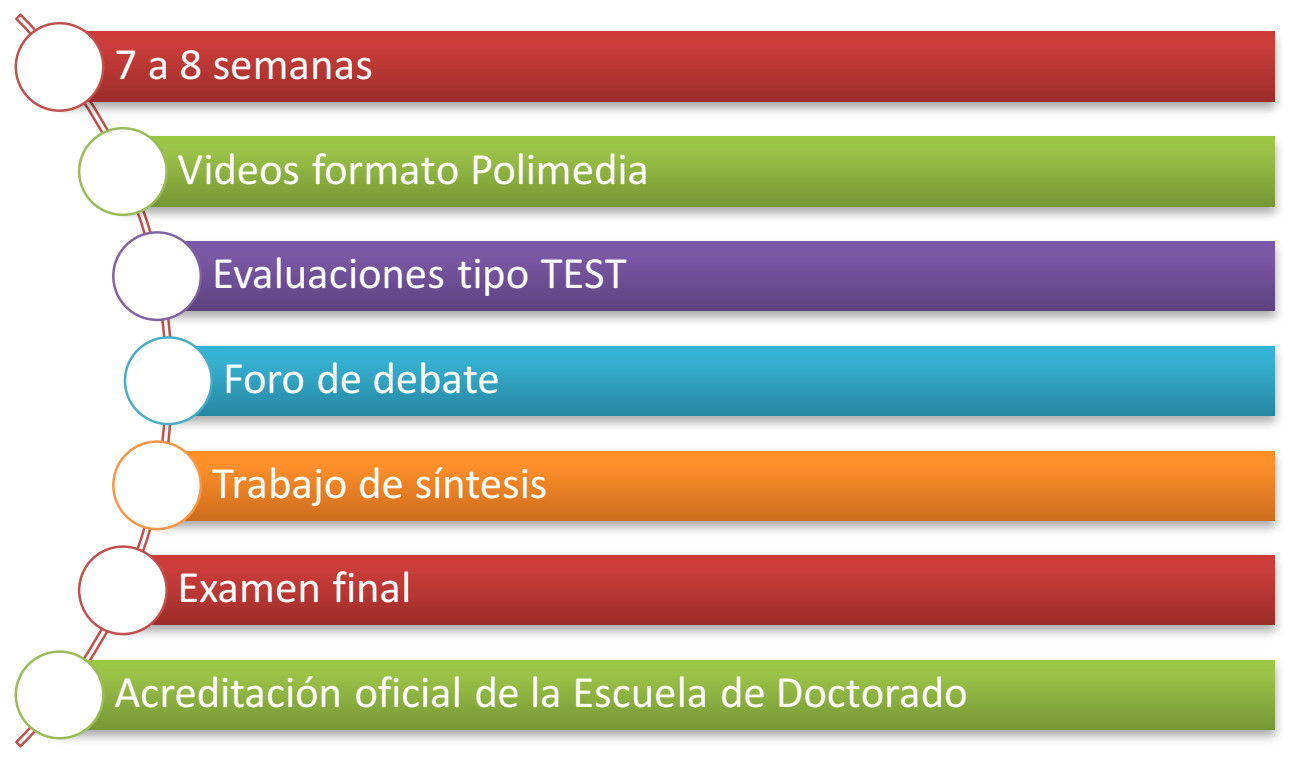

Fig. 1 Características generales del curso

La duración prevista es de 8 semanas. El Curso está disponible en PoliformaT.

En el apartado GUÍA DOCENTE se pueden consultar los objetivos generales, bibliografía, evaluación y un resumen de la ficha docente. El temario está disponible en CONTENIDOS. El material complementario está disponible en RECURSOS. Las pruebas de autoevaluación están en EXÁMENES.

La metodología del curso es ONLINE. Cada uno de los 7 temas contiene:

- Guía de aprendizaje

- Videos con los contenidos

- Recursos adicionales

- Prueba de autoevaluación tipo Test

Adicionalmente se propone la realización de un pequeño trabajo y una prueba de evaluación final de forma online.

El trabajo está publicado en TAREAS. Se plantea como alternativa la participación en el foro de la asignatura.

La prueba objetiva tipo test final se basa en el tipo de preguntas realizadas en las pruebas de autoevaluación. Se utiliza un procedimiento que garantice la identidad de la persona que realice el examen.

\section{(c)) EY-NC-ND 2016, Universitat Politècnica de València}


Se plantea como alternativa a la realización del trabajo, la participación en los foros de debate.

Para conseguir la máxima puntuación en los foros será necesario realizar 10 contribuciones a los foros, con información y comentarios relevantes.

La calificación se basa en el número de contribuciones, la calidad y el porcentaje de lectura de los mensajes de los compañeros:

- Tres contribuciones personales sobre los temas del curso con un mínimo de 200 palabras cada una.

- Siete comentarios a contribuciones de los demás alumnos.

- Se tendrá en cuenta además la lectura de los debates. Para ello será necesario marcar como leídos los mensajes que se hayan visto.

Esta contribución en los foros es opcional, pero se recomienda la participación, como alternativa al trabajo final.

Al finalizar el primer año de impartición se realizó una encuesta a todos los alumnos, en el momento del examen final presencial y se obtuvieron las siguientes conclusiones:

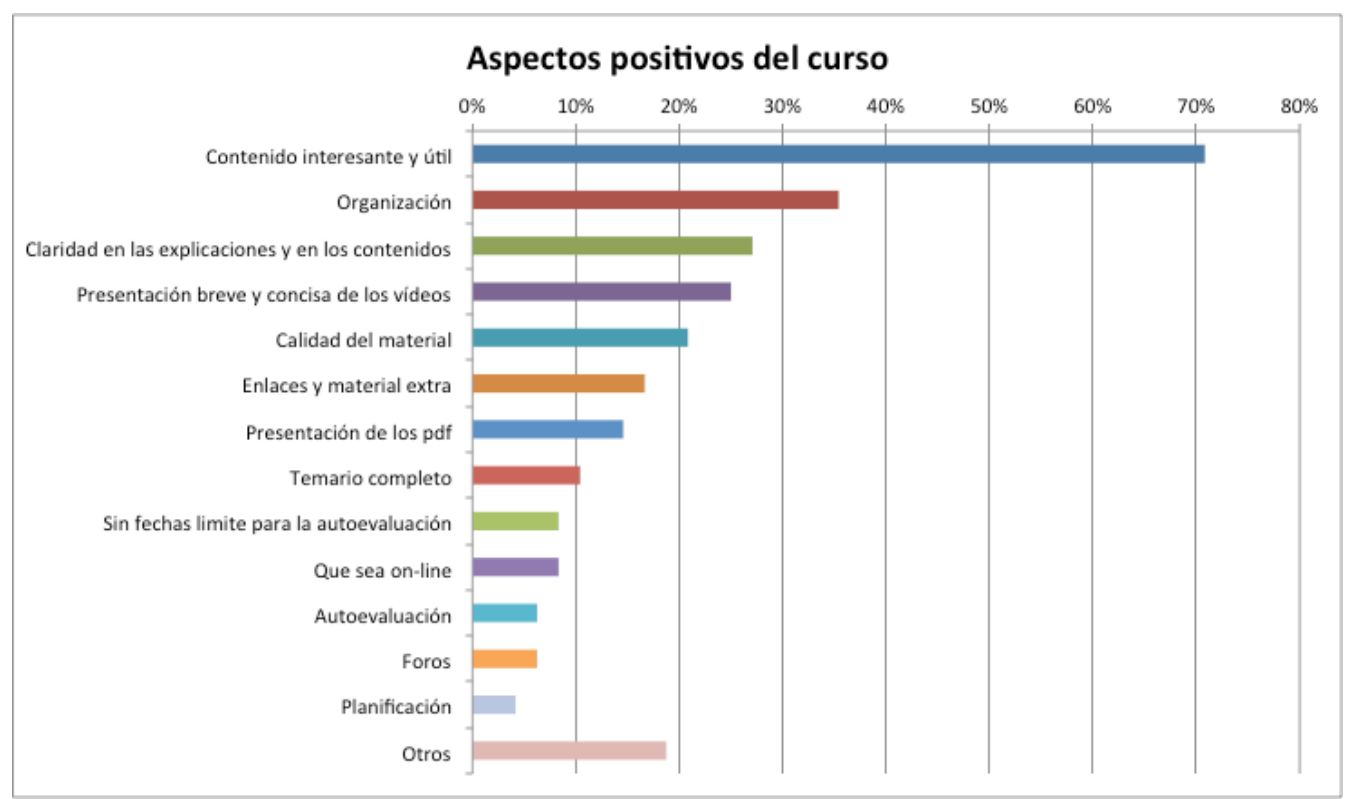

Fig. 2 Encuesta sobre aspectos positivos del curso "Carrera Investigadora" 2013-14

Lo importante en este caso es la identificación de los puntos de mejora necesarios. En la siguiente tabla se observan estos resultados. 


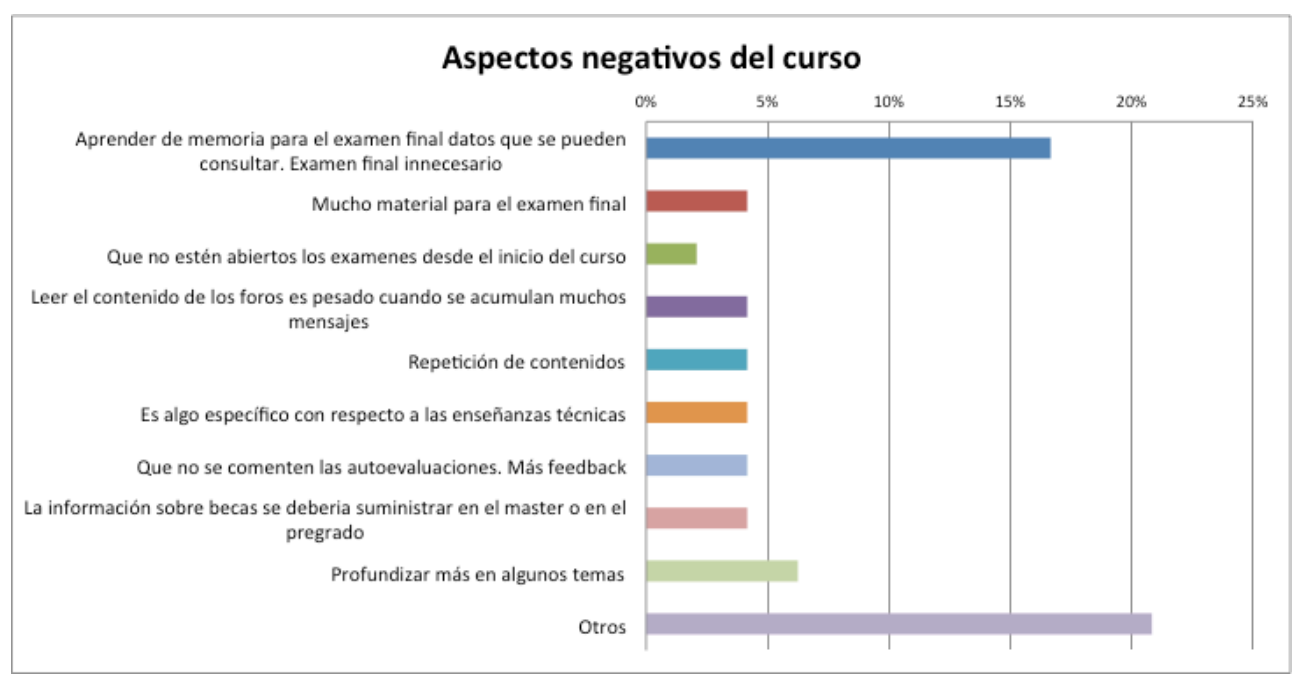

Fig. 3 Encuesta sobre aspectos negativos del curso "Carrera Investigadora" 2013-14

En resumen, los alumnos valoran muy positivamente los contenidos, y la metodología, y la evaluación. Las sugerencias de mejora son diversas y apuntan en diversas direcciones, pero destacan especialmente las que proponen evitar el examen final presencial. Con respecto a los aspectos valorados positivamente, había una valoración relativamente baja para los foros, pese a que la percepción para los profesores era buena.

\section{Desarrollo de la innovación}

Las mejoras propuestas para las siguientes ediciones tuvieron en cuenta los comentarios de los alumnos.

En primer lugar se propuso a la Escuela de Doctorado transformar la evaluación final presencial en evaluación final online, manteniendo la evaluación continua después de cada tema.

Durante el primer año se había planteado la opción de limitar el tiempo de evaluación para cada tema, para forzar el seguimiento semanal de impartición, pero finalmente se decidió ir abriendo progresivamente los contenidos semana a semana, pero quedando abiertos y dejando la evaluación del tema abierta hasta el final del curso. Esta opción fue muy bien valorada por los alumnos, en comparación con otros cursos que forzaban el ritmo de exámenes.

La segunda propuesta de mejora fue el cambio en el sistema de evaluación, incentivando la participación en los foros, que se convirtieron en una vía alternativa al trabajo final. Esta vía ha resultado muy valiosa para las siguientes ediciones.

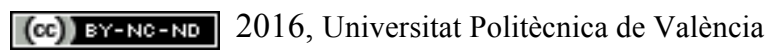

Congreso In-Red (2016) 


\section{Resultados}

Los resultados obtenidos en las tres ediciones han sido las siguientes:

\begin{tabular}{|l|l|l|l|l|}
\hline Curso & Matriculados & Aptos & Suspensos & No presentados \\
\hline $2013-14$ & 90 & 57 & 10 & 23 \\
\hline $2014-15$ & 110 & 101 & 0 & 9 \\
\hline $1015-16$ & 201 & & & \\
\hline
\end{tabular}

El curso "Carrera Investigadora" está siendo muy bien valorado en las encuestas realizadas por la UPV. Hay que indicar que el tipo de encuesta para curso online no es la realizada con carácter general para otras asignaturas. El modelo de encuesta no ha sido aprobado por el Consejo de Gobierno, por lo que sus resultados no se están aplicando al cálculo del Índice de la Actividad Docente, pese a tener un número muy significativo de respuestas con valoración muy positiva.

Durante la última edición del curso, los aspectos positivos más destacados por los alumnos en las encuestas realizadas han sido los siguientes:

- Muy buena organización del curso

- Muy interesante la participación en los foros, ya que permite conocer las experiencias de otros alumnos y completar la información de los temas con conocimientos/experiencias de otros compañeros

- Flexibilidad del curso, al ser on-line

- Buen aprovechamiento de la plataforma PoliformaT

- Material interesante y útil para la carrera investigadora

- Buena comunicación con los profesores

- Positivo poder sustituir el examen final por una participación activa en los foros

En cuanto a los aspectos negativos, algunos alumnos comentan la necesidad de actualizar alguna información, y la necesidad de internacionalizar los contenidos realizando el material y los videos en inglés.

La actualización de información en cursos con contenidos cambiantes de año en año es necesaria cada cierto tiempo, lo que supone un esfuerzo añadido para el profesorado, ya que como mucho es posible aprovechar completamente la información y los videos en un par de ediciones del curso. La actualización de los vídeos Polimedia es por tanto necesaria cada pocos años.

A continuación se muestran en la Fig. 4 algunos de los resultados más destacables de las encuestas del curso 2015-16. 

temporal.
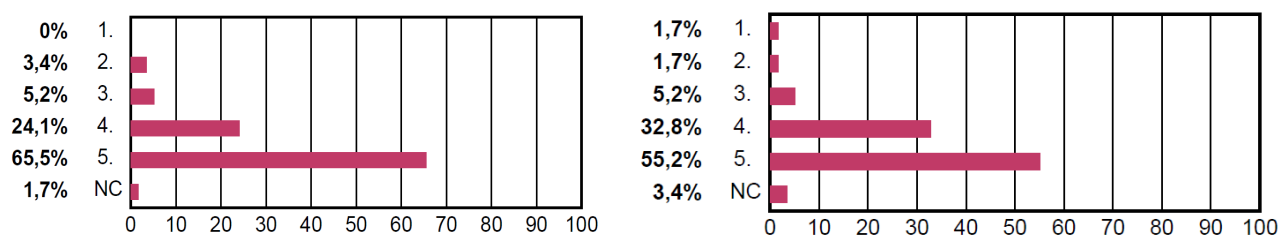

La formación recibida está completamente relacionada con $\mathrm{mi}$

formación transversal en investigación.

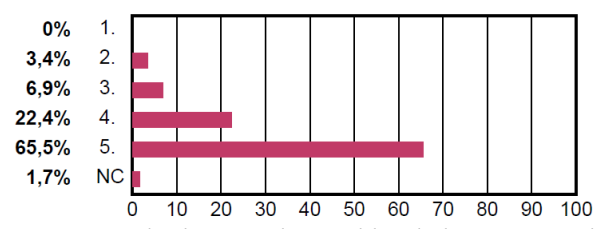

Mis expectativas ante el curso han quedado cubiertas y, en general, estoy satisfecho con el resultado.

Fig. 4 Resultados más destacables de la encuesta del curso "Carrera Investigadora" 2015-16 (1. Totalmente en desacuerdo; 2: Más bien en desacuerdo; 3: Término medio; 4:Más bien de acuerdo; 5: Totalmente de acuerdo; NC: Sin información suficiente).

El número de alumnos se ha duplicado en el curso actual, con un total de 4 grupos de 50 alumnos, debido a que se ha ido conociendo la gran utilidad de los contenidos.

Destacaríamos de forma muy especial el gran éxito obtenido en los foros.Se ha planteado una serie de temas de debate y la participación ha sido la siguiente:

- Evaluación de la investigación ( 255 mensajes)

- Acceso a plazas de profesorado universitario ( 189 mensajes)

- Movilidad y estancias internacionales ( 246 mensajes)

- El doctorado ( 392 mensajes)

- Tipos de contratos de investigación y carrera del investigador ( 316 mensajes)

- Estado actual de la I+D+i en España y en la UPV ( 412 mensajes)

En las tres ediciones realizadas se han ido observando los siguientes hechos:

- Hay un porcentaje de alumnos, que se puede estimar en torno al 50\%, que siguen el ritmo fijado, intentan realizar las pruebas de evaluación en un tiempo no superior a las dos semanas desde la apertura del examen y que participan activamente en los foros.

- Un porcentaje de alumnos estimado en el $20 \%$ que siguen el curso con retraso, pero que completan razonablemente bien las tareas y participación en los foros.

- El $15 \%$ de alumnos dejan para el último momento completar todas las tareas pendientes. 
- Se produjo un abandono del $20 \%$ el primer año, que se ha conseguido bajar al $10 \%$ en la segunda edición.

Una buena muestra puede ser la situación del presente curso académico. La fecha límite que se ha fijado para completar todas las actividades es el día 1 de Abril. En las diversas columnas se muestra el progreso de las unidades completadas. La última columna corresponde al día 27 de Marzo, a 4 días del límite para completar todas las actividades.

\begin{tabular}{|l|l|l|l|l|}
\hline ACTIVIDAD PROPUESTA & Peso & $2^{\text {a }}$ sem. & $4^{\text {a }}$ sem. & $8^{\text {a }}$ sem. \\
\hline Unidad 1 & $5 \%$ & 98 & 125 & 167 \\
\hline Unidad 2 & $5 \%$ & & 102 & 162 \\
\hline Unidad 3 & $5 \%$ & & 51 & 158 \\
\hline Unidad 4 & $5 \%$ & & & 155 \\
\hline Unidad 5 & $5 \%$ & & & 146 \\
\hline Unidad 6 & $5 \%$ & & & 130 \\
\hline Unidad 7 & $5 \%$ & & & 123 \\
\hline Foros & $30 \%$ & & 30 & 72 \\
\hline Tarea & $30 \%$ & & 0 & 15 \\
\hline
\end{tabular}

En la presentación final de la comunicación se podrán mostrar los resultados finales del presente curso académico, una vez realizados los exámenes y entregadas las tareas pendientes.

\section{Conclusiones}

Se puede concluir que los resultados obtenidos en el Curso Online en los últimos años son muy positivos. Se ha conseguido un buen equilibrio entre la actividad de los alumnos sobre todo en los foros. Los resultados demuestran un alto porcentaje de alumnos que completan todas las actividades, que se puede estimar en el $85 \%$, que es un resultado muy notable comparado con el 15\% que se consigue obtener en los cursos MOOC. La organización y modelo pedagógico del curso puede servir como modelo para nuevos cursos similares de nivel universitario. 


\section{Referencias}

BUSQUETS, J., et al. PoliformaT: una estrategia para la formación on-line en la Educación Superior. Virtual Educa 2006, 2006, p. 20-23.

TURRO, Carlos; CAÑERO, Aristóteles; BUSQUETS, Jaime. Video learning objects creation with polimedia. En Multimedia (ISM), 2010 IEEE International Symposium on. IEEE, 2010. p. 371-376.

MENGOD, R. Poliformat, the Sakai-based on-line campus for UPV-history of a success. En 5th Sakai Conference, Vancouver, BC, Canada. 2006.

MARTÍNEZ NAHARRO, Susana, et al. "Los objetos de aprendizaje como recurso de calidad para la docencia: criterios de validación de objetos en la Universidad Politécnica de Valencia". En IV Simposio Pluridisciplinar sobre Diseño y Evaluación de Contenidos Educativos Reutilizables (SPDECE 2007). 2007

SIMÓ TEN, José Enrique, et al. Jornadas de Innovación Educativa y Docencia en Red de la Universitat Politècnica de València. 2014. 